\section{Performance of GDx VCC in eyes with peripapillary atrophy: comparison of three circle sizes}

\begin{abstract}
Purpose A scanning laser polarimetry (GDx VCC) equips three different sized measurement circles. In eyes with peripapillary atrophy (PPA), the GDx measurement becomes inaccurate when the circle falls on PPA. The aim of this study was to evaluate performance of the three circles of GDx measurement in eyes with PPA.

Methods Three different sized circles were compared regarding frequency of PPA, which fell on each circle in 282 open-angle glaucoma (OAG) eyes, reproducibility of GDx parameters in 24 normal and 22 OAG eyes, and ability to detect glaucoma in 50 normal and 50 OAG eyes. Results PPA was observed in $230(82 \%)$ of 282 OAG eyes. PPA fell on the small circle (default setting), medium, and large circles in 119 $(43 \%), 38(14 \%)$, and $12(4 \%)$ of the 280 OAG eyes. Reproducibility of GDx parameters was not significantly different among three circles in normal eyes $(P>0.05)$, whereas coefficients of reproducibility of TSNIT average $(P=0.006)$ and superior average $(P=0.035)$ were smaller in the smaller circles in OAG eyes. GDx parameters significantly correlated $(P<0.001)$, but were significantly different $(P<0.05)$ between the small and medium circles. The area under receiver operating characteristic curves for dividing OAG from normal eyes using GDx parameters was similar between the small and medium circles.

Conclusions If the medium circles were used, obstructing influences of PPA on GDx measurement could be avoided more often in Japanese OAG eyes with similar reproducibility and comparable ability to detect glaucoma compared to those with the default small circle.
\end{abstract}

Eye (2008) 22, 173-178; doi:10.1038/sj.eye.6702516; published online 4 August 2006
S Kunimatsu, A Tomidokoro, H Saito, M Aihara, $\mathrm{G}$ Tomita and M Araie

Keywords: peripapillary atrophy; GDx; VCC; open-angle glaucoma; scanning laser polarimetry

\section{Introduction}

Accuracy of measurements of the retinal nerve fiber layer (RNFL) thickness using a scanning laser polarimetry (SLP), GDx (Carl Zeiss Meditec, Dublin, CA, USA), has been improved after the introduction of the variable corneal compensation (VCC) system, which reduces the influence of the anterior segment birefringence in an individual eye. ${ }^{1-4}$ However, one of the remaining drawbacks of SLP is that RNFL measurements are inaccurate when peripapillary atrophy (PPA) fell on the measurement circle (Figure 1). As PPA is more frequent in myopic eyes ${ }^{5,6}$ and myopia is much more common among Japanese, ${ }^{7}$ it is supposed that PPA is commonly accompanied with Japanese patients of open-angle glaucoma (OAG).

With new software (GDx VCC version 5.3.2.), two different sizes of circles (medium and large) are available in addition to the default size circle (small). The inner diameters are 2.4, 3.2, and $4.0 \mathrm{~mm}$ for small, medium, and large circles, respectively, in eyes with normal refraction, although the actual sizes will be altered according to the refractive error of each eye. Using the medium or large circle instead of the default small circle, the obstructing influence of PPA on the RNFL measurement would be often avoided. To our knowledge, however, no report is available on the clinical usefulness of the medium or large circle. The aim of this study was to estimate the frequency of PPA in Japanese OAG eyes and to assess the reliability and usefulness of the RNFL measurements
Department of Ophthalmology, University of Tokyo Graduate School of Medicine, Tokyo, Japan

Correspondence:

A Tomidokoro,

Department of

Ophthalmology,

University of Tokyo

Graduate School

of Medicine,

7-3-1 Hongo, Bunkyo-ku, Tokyo 113-8655,

Japan

Tel: + 81338155411 .

Fax: + 81338970798

E-mail: tomidokoro-tky@ umin.ac.jp

Received: 26 January 2006 Accepted: 12 June 2006 Published online: 4 August 2006 

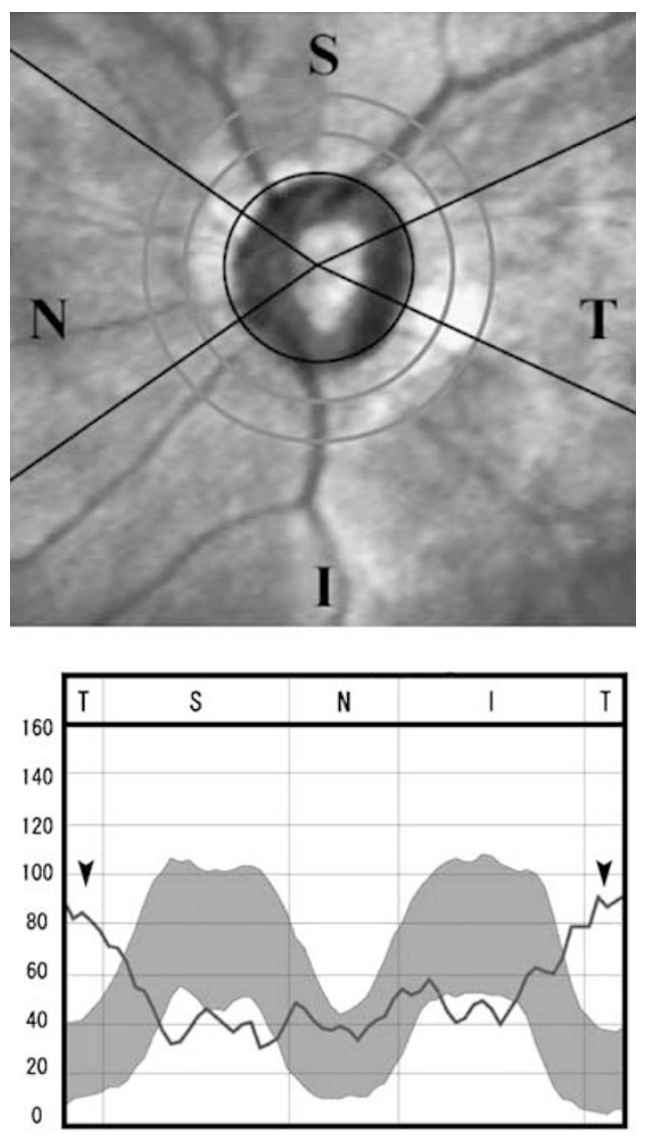

Figure 1 An example of GDx VCC measurements in eyes with PPA. This eye has a large PPA and the temporal portion of the PPA falls on the measurement circle (small circle) (a). As the consequence, retinal neve fiber layer thickness is measured abnormally thicker in the temporal area (arrowheads, b).

using medium or large circle for those eyes in which the small circle would fall on the area with PPA.

\section{Methods}

The studies below were carried out at the Department of Ophthalmology of the University of Tokyo, Graduate School of Medicine. Written informed consent to use their clinical data for this study was obtained from all subjects. The study was approved by the Institutional Review Board for Human Research of the University of Tokyo, Graduate School of Medicine and was conducted according to the tenets of the Declaration of Helsinki.

\section{Measurement of GDx VCC}

SLP images were obtained using GDx VCC with a software version 5.3.2. The general principles of SLP have been described in detail elsewhere. ${ }^{8,9}$ Imaging was obtained under dim light and with undilated pupils. For each eye, the macula was imaged without compensation, and the eye-specific corneal polarization axis and corneal polarization magnitude were recorded. Corneal birefringence-compensated SLP images then were acquired using the eye-specific corneal polarization axis and magnitude by adjusting the VCC retarder. Determination of the GDx VCC parameters was performed with regard to the small (default), medium, and large circles, respectively, on each acquired image with quality score of 8 or more.

\section{Frequency of PPA falling on circles in OAG eyes}

The frequency of eyes in which PPA fell on each of the GDx circles was determined using the stocked data of 282 eyes of 141 OAG patients continuously obtained between September 2003 and May 2004. A diagnosis of OAG was made according to the typical glaucomatous optic disc findings, the corresponding visual field damage, and the absence of any contributing ocular or systemic disorders in disregard of intraocular pressure (IOP). Eyes with refractive errors (spherical equivalent) larger than $8 \mathrm{D}$ and eyes had history of intraocular surgery including laser were not included. The patients studied included 81 male and 60 female patients and age averaged $55.4 \pm 12.3$ (mean \pm SD) years, refractive error $-3.9 \pm 3.5 \mathrm{D}$, IOP with or without medication $18.3 \pm 3.9 \mathrm{mmHg}$, and mean deviation (MD) of the Humphrey Field Analyzer 30-2 SITA standard (Carl Zeiss Meditec, Dublin, CA, USA) $-6.0 \pm 5.4 \mathrm{~dB}$.

The GDx VCC results of each patient were reloaded from the hard disc drive of the instrument and the small, medium, and large circles were drawn. Beta zone of PPA, which was identified by choroidal atrophy with visible large choroidal vessels and sclera, was determined and judged whether each of the measurement circles fell on the PPA or not on the display of GDx VCC instrument with the help of colour fundus photographs if necessary. Alpha zone of PPA, which located peripherally and characterized by irregular hypopigmentation, hyperpigmentation, or both, was not included in the current study.

\section{Reproducibility of the GDx measurements using the three circles}

Reproducibility of GDx VCC parameters obtained with three circles was evaluated in 24 eyes of 16 ophthalmologically healthy subjects (mean age, $35.1 \pm 6.2$ years) and 22 eyes of 14 patients with OAG (mean age, $53.8 \pm 13.6$ years) in whom the data were newly obtained. The criteria of the diagnosis of OAG were same as above. In the OAG patients, refractive error averaged $-3.3 \pm 3.7 \mathrm{D}$, IOP with or without medication $14.1 \pm 3.1 \mathrm{mmHg}$, and $\mathrm{MD}-5.6 \pm 5.5 \mathrm{~dB}$. 
Ophthalmologically healthy subjects had IOP not exceeding $21 \mathrm{mmHg}$, the optic disc with a normal appearance, a normal open angle, a normal visual field defined, and had no history of ocular diseases or a family history of glaucoma. In the ophthalmologically healthy subjects, refractive error averaged $-1.0 \pm 1.4 \mathrm{D}$.

Each eye was scanned twice on separate days within a 1 -month period. Eyes were excluded when any of the three circles fell on PPA or image quality fell below 8 . The coefficient of the reproducibility for each parameter was calculated as follows: $\left|V_{1}-V_{2}\right| /\left(V_{1}+V_{2}\right) / 2 \times 100 \%$, where $V_{1}$ is the first and $V_{2}$ the second measurement obtained.

\section{Mean values of the GDx parameters and detection of glaucoma}

As the fraction of poor-quality images is rather high for the large circle (see Results section), the performance of the GDx was not determined for this circle size. Comparison of the GDx parameters between 50 ophthalmologically normal eyes of 50 subjects (mean age, $50.6 \pm 8.7$ years) and 50 OAG eyes of 50 patients (mean age, $49.4 \pm 9.1$ years), of whom data were newly obtained for this part of the current study, and ability to detect glaucomatous eyes using GDx VCC was evaluated. The criteria of ophthalmologically healthy subjects and the diagnosis of OAG patients were same as above. In the OAG patients, refractive error averaged $-3.6 \pm 2.5 \mathrm{D}$, IOP with or without medication $14.2 \pm 3.3 \mathrm{mmHg}$, and MD
$-4.2 \pm 2.9 \mathrm{~dB}$. In the ophthalmologically healthy subjects, refractive error averaged $-0.8 \pm 1.6 \mathrm{D}$.

\section{Statistical analysis}

Statistical analyses were performed using a statistical software package, SPSS 13.0J for Windows (SPSS Japan Inc., Tokyo, Japan). Friedman's test was used to compare the averages among three groups. The difference between two groups was evaluated using the Wilcoxon's signed-rank test or the $\chi^{2}$ test. Statistical correlation was evaluated by Spearman's rank correlation coefficient.

Receiver operating characteristic (ROC) curves were used to describe the ability to differentiate OAG eyes from normal eyes. A $P$-value less than 0.05 was considered statistically significant.

\section{Results}

Frequency of PPA falling on circles in OAG eyes

PPA could not be determined owing to expanded chorioretinal atrophy around the optic disc in two eyes. PPA was observed in $230(82 \%)$ of the remaining 280 OAG eyes. PPA fell on the small, medium, and large circles in 119 (43\%), 38 (14\%), and 12 (4\%) of the 280 eyes, respectively. The frequency with small circle was significantly greater than that with medium circle $(P<0.001)$, and that with medium circle was greater than that with large circle $(P<0.001)$. The GDx VCC

Table 1 Coefficient of reproducibility for the GDx parameters obtained with small, medium, and large circles in normal and OAG eyes

\begin{tabular}{|c|c|c|c|c|}
\hline & Small circle & Medium circle & Large circle & P-values* \\
\hline \multicolumn{5}{|c|}{ TSNIT average } \\
\hline Normal & $3.3(1.6,5.1)$ & $4.3(1.9,6.6)$ & $5.0(2.1,8.0)$ & 0.14 \\
\hline $\mathrm{OAG}$ & $4.5(0.7,8.3)$ & $6.2(1.1,11.2)$ & $7.8(2.8,12.9)$ & 0.006 \\
\hline \multicolumn{5}{|c|}{ Superior average } \\
\hline Normal & $3.7(2.3,5.1)$ & $4.6(2.5,6.7)$ & $5.2(2.5,7.9)$ & 0.5 \\
\hline OAG & $2.2(0.7,3.8)$ & $3.1(0.2,6.0)$ & $4.6(1.1,8.1)$ & 0.035 \\
\hline \multicolumn{5}{|c|}{ Inferior average } \\
\hline Normal & $4.6(2.5,6.6)$ & $4.8(2.3,7.3)$ & $5.7(2.9,8.6)$ & 0.080 \\
\hline OAG & $7.5(2.6,12.4)$ & $14.2(1.5,27.0)$ & $14.9(5.5,24.2)$ & 0.078 \\
\hline \multicolumn{5}{|l|}{ TSNIT SD } \\
\hline Normal & $7.4(4.4,10.4)$ & $7.2(4.3,10.0)$ & $7.0(4.1,9.9)$ & 0.9 \\
\hline OAG & $8.2(4.1,12.3)$ & $7.4(3.6,11.1)$ & $10.7(5.8,15.6)$ & 0.060 \\
\hline \multicolumn{5}{|l|}{ NFI } \\
\hline Normal & $13.3(8.2,20.3)$ & $19.5(13.4,29.2)$ & $20.8(10.9,29.1)$ & 0.054 \\
\hline OAG & $17.6(9.0,26.2)$ & $19.4(7.8,30.9)$ & $16.7(5.9,27.5)$ & 0.6 \\
\hline
\end{tabular}

NFI, nerve fiber indicator; OAG, open-angle glaucoma; SD, standard deviation.

Data are shown with $95 \%$ confidence interval in the parentheses.

${ }^{*} P$-value for difference among three circles (Friedman's test). 
parameters could not be obtained in $34(12 \%), 41(15 \%)$, and 71 (25\%) of 282 eyes for the small, medium, and large circles, respectively, because of poor image quality score $(<8)$ or extrusion of the circle from the measurement area. Those frequencies were significantly different among the circles $\left(P<0.001, \chi^{2}\right.$ test $)$ and the frequency with the large circle was significantly larger than that with the small or medium circle $(P<0.001, P=0.002$, respectively).

\section{Reproducibility of the measurements using the three circles}

Coefficients of reproducibility of the GDx parameters in normal or OAG eyes are shown in Table 1 . These were not significantly different among the three circles in normal eyes for each parameter $(P>0.05$, Friedman's test), whereas coefficients of reproducibility of TSNIT average $(P=0.006)$ and superior average $(P=0.035)$ were smaller in the smaller circles in OAG eyes.

\section{Mean values of the GDx parameters and detection of glaucoma}

Mean values of the GDx parameters are shown in Table 2. All parameters in OAG eyes were significantly smaller than in normal eyes $(P<0.05$, Wilcoxon's signed-rank test). Between the small and medium circles, all of the GDx parameters significantly correlated (Spearman's rank correlation coefficients $>0.8, P<0.001$ ).

The ROC curves for dividing OAG eyes from normal eyes using each parameter obtained with the small and medium circles were determined. The area under the ROC curves is shown in Table 3. Ninety-five percent confidence interval was overlapping each other between the small and medium circles, suggesting there was no apparent difference in the ability to detect OAG eyes between these circles. Among the indices, the nerve fiber indicator (NFI) had the largest area under the ROC curve. ROC curves regarding TSNIT average and NFI are drawn in Figure 2.

Table 3 Area under the ROC for dividing glaucomatous from normal eyes using the small and medium circles

\begin{tabular}{lcc}
\hline & Small circle & Medium circle \\
\hline TSNIT average & $0.90(0.85,0.96)$ & $0.83(0.74,0.91)$ \\
Superior average & $0.86(0.78,0.93)$ & $0.86(0.78,0.93)$ \\
Inferior average & $0.91(0.86,0.97)$ & $0.85(0.78,0.92)$ \\
TINIT SD & $0.80(0.71,0.88)$ & $0.84(0.76,0.91)$ \\
NFI & $0.94(0.90,0.99)$ & $0.93(0.88,0.98)$
\end{tabular}

$\mathrm{NFI}$, nerve fiber indicator; ROC, receiver operating curve; SD, standard deviation.

Data (area under the ROC) are shown with 95\% confidence interval in the parentheses.

Table 2 Differences and correlation of the GDx parameters between normal and OAG eyes

\begin{tabular}{|c|c|c|c|c|c|c|}
\hline & \multicolumn{2}{|c|}{ Small circle } & \multicolumn{2}{|c|}{ Medium circle } & \multicolumn{2}{|c|}{ Small vs medium circles } \\
\hline & Mean $(95 \%$ CI $)$ & P-values ${ }^{\mathrm{a}}$ & Mean $(95 \%$ CI $)$ & $\mathrm{P}$-values ${ }^{\mathrm{a}}$ & Correlation (P-value) & P-values ${ }^{\mathrm{b}}$ \\
\hline \multicolumn{7}{|c|}{ TSNIT average } \\
\hline Normal & $56(54,57)$ & \multirow{2}{*}{$<0.001$} & $49(48,51)$ & \multirow{2}{*}{$<0.001$} & $0.89(<0.001)$ & $<0.001$ \\
\hline OAG & $46(44,48)$ & & $42(40,43)$ & & $0.86(<0.001)$ & $<0.001$ \\
\hline \multicolumn{7}{|c|}{ Superior average } \\
\hline Normal & $68(67,70)$ & \multirow{2}{*}{$<0.001$} & $59(58,61)$ & \multirow{2}{*}{$<0.001$} & $0.89(<0.001)$ & $<0.001$ \\
\hline OAG & $55(52,59)$ & & $48(45,50)$ & & $0.93(<0.001)$ & $<0.001$ \\
\hline \multicolumn{7}{|c|}{ Inferior average } \\
\hline Normal & $66(63,68)$ & \multirow{2}{*}{$<0.001$} & $59(57,61)$ & \multirow{2}{*}{$<0.001$} & $0.94(<0.001)$ & $<0.001$ \\
\hline OAG & $49(46,53)$ & & $47(44,50)$ & & $0.85(<0.001)$ & $<0.001$ \\
\hline \multicolumn{7}{|l|}{ TSNIT SD } \\
\hline Normal & $23(22,24)$ & \multirow{2}{*}{$<0.001$} & $21(20,23)$ & \multirow{2}{*}{$<0.001$} & $0.96(<0.001)$ & $<0.001$ \\
\hline OAG & $19(16,22)$ & & $16(15,17)$ & & $0.90(<0.001)$ & $<0.001$ \\
\hline \multicolumn{7}{|l|}{ NFI } \\
\hline Normal & $18(16,20)$ & \multirow{2}{*}{$<0.001$} & $17(15,18)$ & \multirow{2}{*}{$<0.001$} & $0.82(<0.001)$ & 0.027 \\
\hline OAG & $44(39,49)$ & & $42(37,47)$ & & $0.82(<0.001)$ & 0.008 \\
\hline
\end{tabular}

$\mathrm{CI}$, confidence interval; NFI, nerve fiber indicator; SD, standard deviation.

Data are shown with $95 \%$ confidence interval $(95 \% \mathrm{CI})$ in the parentheses.

${ }^{a} P$-value for the difference of the means between normal and OAG eyes (Mann-Whitney $U$-test). Correlation $=$ Spearman's rank correlation coefficient (with $P$-value) between small and medium circles.

${ }^{\mathrm{b}} P$-value for the difference of means between small and medium circles (Wilcoxon's signed-rank test). 


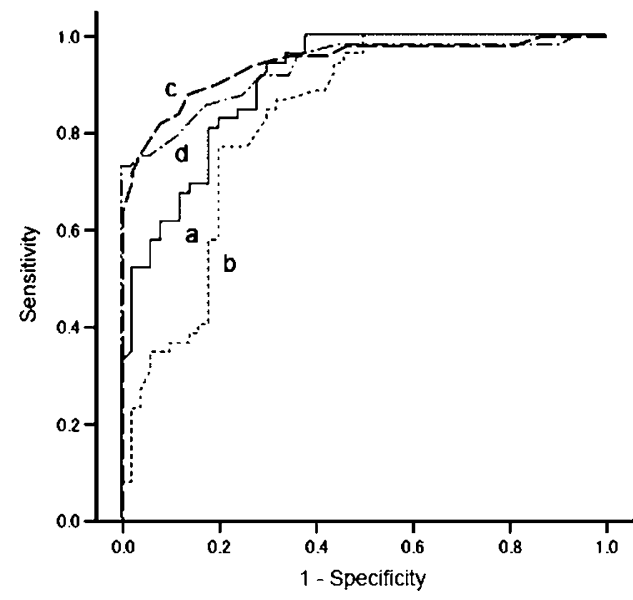

Figure 2 The ROC curves for discriminating between normal and glaucomatous eyes regarding TSNIT average of the small circle (a), that of the medium circle (b), the NFI of the small circle (c), and that of the medium circle (d).

\section{Discussion}

\section{Frequency of PPA falling on circles on OAG eyes}

The presence of PPA reduces the accuracy of the RNFL measurement using a SLP. Bowd et al ${ }^{4}$ reported that in five of $73(7 \%)$ eyes the circle fell on PPA and they used the expanded circle ( $20 \%$ horizontally and $20 \%$ vertically) for the analysis of those eyes. PPA is more frequently and more extensively observed in patients with glaucoma than in normal subjects in Caucasians ${ }^{10,11}$ (no data available in Japanese) and associated with glaucomatous damage. ${ }^{12-14}$ Because myopia is more likely associated with PPA, ${ }^{5,6}$ and more frequent in Japan, ${ }^{7}$ the influence of PPA on the measurement of the RNFL is of practical concern, especially in Japanese patients with OAG. In the current study, PPA fell on the small circle (default setting) in 119 (43\%) of 280 OAG eyes, suggesting that accurate GDx measurement may be often disturbed because of PPA in Japanese OAG eyes when using the small circle.

When using optical coherence tomography (OCT), Lai et $a l^{15}$ reported good agreement between the automated and manual tracing results in eyes with PPA, and the authors concluded that automated optic nerve head analysis using OCT may be used in the clinical setting in eyes with PPA. In contrast, the accuracy of GDx VCC when measuring the RNFL thickness decreases in the presence of PPA (Figure 1), and alternative measurement methods should have clinical value.

In the current study, the GDx parameters for the small, medium, and large circles could not be obtained in $34(12 \%), 41(15 \%)$, and $71(25 \%)$ of 282 OAG eyes, respectively, because of poor image quality score $(<8)$ or deviancy of the circle from the measurement area. Unless the optic disc was successfully located at the centre of the measurement area, the large circle easily extrude from the area. Moreover, the measurement using the large circle should be unstable because of the influence of the thinner RNFL, which was reported to often show atypical retardation pattern (ARP). ${ }^{16-18}$

\section{Reproducibility of the measurements using the three circles}

Good intraoperator and interoperator measurement reproducibility using an SLP has been reported by several investigators. ${ }^{19-21}$ In the present study, we evaluated the difference in intraoperator reproducibility among the circles (Table 1). The coefficients of reproducibility in normal eyes were $8 \%$ or less, except $\mathrm{NFI}$, and there was no significant difference among the three different sized circles. On the other hand, in the glaucomatous eyes, the reproducibility of some parameters was worse for the larger circles than the small ones. The sensitivity of GDx VCC is reported to be reduced in the retinal area with ARP. ${ }^{16-18}$ This should be at least partially responsible for the poor reproducibility of the measurements with the outer circles. And besides, the influence of ARP owing to the outer and thinner retinal area should be involved with the performance in diagnosing glaucoma or assessing glaucoma progression described below.

\section{Mean values of the GDx parameters and detection of glaucoma}

Varma $e a^{22}$ measured histologically the thickness of the RNFL in normal human eyes and reported that the thickness decreased with increasing distance from the disc margin. In this study, the RNFL thickness obtained with the small circle was greater than that with the medium circle in normal and glaucomatous eyes, which is consistent with histologic results.

To determine the ability of each parameter to differentiate glaucomatous from normal eyes, we analysed the ROC curves. The largest area under the curve (AUC) was the NFI (0.94 with the small circle, 0.93 with the medium circle), which was similar to a previous study. Medeiros $e a^{23}$ reported that the AUC for NFI using GDx VCC was 0.91. There was no apparent difference between the small and medium circles when differentiating glaucoma from normal eyes. In the present version of GDx VCC, the NFI has been trained on data measured along the small circle. If a newly trained $\mathrm{NFI}$, which is specially designed for the medium circle, is available, performance to detect glaucoma using the medium circle would be improved. Moreover, the 
potential for the assessment of glaucoma progression may differ among the different circle sizes.

Not only beta zone of PPA but also alpha zone often shows ARP and can assert influence on GDx VCC measurements. However, because the precise determination of alpha area was difficult in some of the eyes as reported previously, ${ }^{14}$ alpha area was not included in the present analyses. Therefore, in the current study, even if the larger circles were used and beta zone was not involved in the SLP measurement area, the disturbance of alpha zone of PPA might be still remained. Recently, a new software version, named the enhanced corneal compensation (ECC), was published. ${ }^{16-18}$ It is reported to significantly diminish the artefacts owing to ARP. This new software may improve the performance of GDx in eyes with PPA even when using the small circle. However, whether the influence of PPA can be completely diminished with ECC is still unclear and further investigation on ECC measurements using the different measurement circles should deserve future investigation.

In conclusion, measurements with the medium circle may be a useful alternative, especially in Japan ${ }^{7}$ and other East-Asian countries where the prevalence of myopia with PPA is higher than in Western countries. ${ }^{24,25}$

\section{References}

1 Weinreb RN, Bowd C, Zangwill LM. Glaucoma detection using scanning laser polarimetry with variable corneal polarization compensation. Arch Ophthalmol 2002; 120: 218-224.

2 Greenfield DS, Knighton RW, Feuer WJ, Schiffman JC, Zangwill L, Weinreb RN. Correction for corneal polarization axis improves the discriminating power of scanning laser polarimetry. Am J Ophthalmol 2002; 134: 27-33.

3 Bagga H, Greenfield DS, Feuer W, Knighton RW. Scanning laser polarimetry with variable corneal compensation and optical coherence tomography in normal and glaucomatous eyes. Am J Ophthalmol 2003; 135: 521-529.

4 Bowd C, Zangwill LM, Weinreb RN. Association between scanning laser polarimetry measurements using variable corneal polarization compensation and visual field sensitivity in glaucomatous eyes. Arch Ophthalmol 2003; 121: 961-966.

5 Ramrattan RS, Wolfs RC, Jonas JB, Hofman A, de Jong PT. Determinants of optic disc characteristics in a general population: The Rotterdam Study. Ophthalmology 1999; 106: 1588-1596.

6 Vongphanit J, Mitchell P, Wang JJ. Population prevalence of tilted optic disks and the relationship of this sign to refractive error. Am J Ophthalmol 2002; 133: 679-685.

7 Shiose Y, Kitazawa Y, Tsukahara S, Akamatsu T, Mizokami $\mathrm{K}$, Futa R et al. Epidemiology of glaucoma in Japan: a nationwide glaucoma survey. Jpn J Ophthalmol 1991; 35: 133-135.
8 Weinreb RN, Shakiba S, Zangwill L. Scanning laser polarimetry to measure the nerve fiber layer of normal and glaucomatous eyes. Am J Ophthalmol 1995; 119: 627-636.

9 Zhou Q, Weinreb RN. Individualized compensation of anterior segment birefringence during scanning laser polarimetry. Invest Ophthalmol Vis Sci 2002; 43: 2221-2228.

10 Jonas JB, Nguyen XN, Gusek GC, Naumann GO. Peripapillary chorioretinal atrophy in normal and glaucoma eyes. I. Morphometric data. Invest Ophthalmol Vis Sci 1989; 5: 908-918.

11 Jonas JB, Konigsreuther KA, Naumann GO. Optic disc histomorphometry in normal eyes and eyes with secondary angle-closure glaucoma. II. Peripapillary region. Graefe's Arch Clin Exp Ophthalmol 1992; 230: 134-139.

12 Jonas JB, Naumann GO. Peripapillary chorioretinal atrophy in normal and glaucoma eyes. II. Correlations. Invest Ophthalmol Vis Sci 1989; 30: 919-926.

13 Jonas JB, Fernandez MC, Naumann GO. Glaucomatous peripapillary atrophy. Occurrence and correlations. Arch Ophthalmol 1992; 110: 214-222.

14 Park KH, Tomita G, Liou SY, Kitazawa Y. Correlation between peripapillary atrophy and optic nerve damage in normal-tension glaucoma. Ophthalmology 1996; 103: 1899-1906.

15 Lai E, Wollstein G, Price LL, Paunescu LA, Stark PC, Fujimoto JG et al. Optical coherence tomography disc assessment in optic nerves with peripapillary atrophy. Ophthalmic Surg Lasers Imag 2003; 34: 498-504.

16 Toth M, Hollo G. Evaluation of enhanced corneal compensation in scanning laser polarimetry: comparison with variable corneal compensation on human eyes undergoing LASIK. J Glaucoma 2006; 15: 53-59.

17 Toth M, Hollo G. Enhanced corneal compensation for scanning laser polarimetry on eyes with atypical polarisation pattern. Br J Ophthalmol 2005; 89: 1139-1142.

18 Sehi M, Guaqueta DC, Greenfield DS. An enhancement module to improve the atypical birefringence pattern using scanning laser polarimetry with variable corneal compensation. Br J Ophthalmol 2006; 90: 749-753.

19 Hoh ST, Ishikawa H, Greenfield DS, Liebmann JM, Chew SJ, Ritch R. Peripapillary nerve fiber layer thickness measurement reproducibility using scanning laser polarimetry. J Glaucoma 1998; 7: 12-15.

20 Kook MS, Sung K, Park RH, Kim KR, Kim ST, Kang W. Reproducibility of scanning laser polarimetry (GDx) of peripapillary retinal nerve fiber layer thickness in normal subjects. Graefe's Arch Clin Exp Ophthalmol 2001; 239: 118121.

21 Rhee DJ, Greenfield DS, Chen PP, Schiffman J. Reproducibility of retinal nerve fiber layer thickness measurements using scanning laser polarimetry in pseudophakic eyes. Ophthalmic Surg Lasers 2002; 33: 117-122.

22 Varma R, Skaf M, Barron E. Retinal nerve fiber layer thickness in normal human eyes. Ophthalmology 1996; 103: 2114-2119.

23 Medeiros FA, Zangwill LM, Bowd C, Weinreb RN. Comparison of the GDx VCC scanning laser polarimeter, HRT II confocal scanning laser ophthalmoscope, and stratus OCT optical coherence tomograph for the detection of glaucoma. Arch Ophthalmol 2004; 122: 827-837.

24 Mutti DO, Bullimore MA. Myopia: an epidemic of possibilities? Optom Vis Sci 1999; 76: 257-258.

25 Saw SM, Katz J, Schein OD, Chew SJ, Chan TK. Epidemiology of myopia. Epidemiol Rev 1996; 18: 175-187. 